\title{
Descripción del fenómeno delictivo en la ciudad de Murcia a partir de herramientas SIG
}

\author{
Description of criminal phenomenon in the city of Murcia using GIS tools
}

\author{
José Antonio Albaladejo-García ${ }^{1}$ \\ Marina Campos-Cotanda ${ }^{2}$
}

\section{Resumen}

Los mapas del delito elaborados a partir de herramientas SIG tienen un papel fundamental para la representación de los fenómenos delictivos, los cuales no se distribuyen de forma homogénea en el espacio ni en el tiempo, permitiendo la observación e interpretación de las conductas delictivas. Estas conductas han sido objeto de investigación a lo largo de los años por la Criminología Ambiental, que ha tenido como objetivo la búsqueda del dónde y cuándo sucede el crimen. En este contexto, la delincuencia tiene un claro carácter geográfico, por lo que más que una explicación aleatoria y de azar, estaría explicada por la georreferenciación de los delitos, posibilitando a su vez métodos no sólo de análisis, sino también de predicción y prevención. Así pues, los denominados "hot spots" adquieren un carácter vinculante en diferentes organismos y plataformas policiales, logrando la visualización espacial y temporal de los hechos delictivos de una determinada región. Este tipo de análisis espacial se llevará a cabo para la ciudad de Murcia y sus 28 barrios, que constituyen el núcleo principal de la Región de Murcia.

Palabras clave: Criminología ambiental; Geografía social; Hot spots; Sistemas de información geográfica (SIG); Mapas del delito.

\begin{abstract}
The crime maps produced from GIS tools have a key role in representing criminal phenomena that are not distributed evenly in space or in time. These maps mean that criminal behavior can be observed and interpreted, and they give the operator many system variables with spatial information. These behaviors have been investigated over the years by the Environmental Criminology, whose objective has been to find out where and when the crime happens. In this context, crime has a clear geographical nature; it's more than just a random explanation. It could be explained by the georeferencing of crimes, which in turn means that not only analysis methods but also prediction and crime prevention methods can be used. The so-called "hot spots" become essential in different institutions and police platforms, so that the crimes committed in a particular region are displayed in time and space. This type of spatial analysis will be conducted for the city of Murcia and its 28 districts, which make up the core of the Murcia Region.
\end{abstract}

Keywords: Environmental criminology; Social Geography; Hot spots; Geographic Information Systems (GIS); Crime maps.

\section{Introducción}

El objetivo de la Criminología es estudiar el fenómeno delictivo, a los actores del mismo (víctima y victimario) y el control social del delito. Tradicionalmente, la Criminología Clásica estudió con profundi-

\footnotetext{
1 Departamento de Geografía. Universidad de Murcia. joseantonio.albaladejo@um.es

2 Departamento de Criminología. Universidad de Murcia. Marina.inves@gmail.com
} 
dad la criminalidad desde diversas perspectivas (biológicas, psicológicas, sociológicas...) que intentaron dar explicación del comportamiento de los individuos infractores y sus motivaciones. Según Sherman, Gartin \& Buerguer (1989), criminólogos como Jeffery, Jacobs o Newman denunciaron, a comienzos de los años 70 , el fracaso del sistema social, judicial, y policial, ofreciendo alternativas que destacaran la modificación del ambiente y no tanto la figura del delincuente (Ariza, 2011).

A raíz de estos planteamientos surgió la Criminología Ambiental que se define como un conjunto de teorías criminológicas que resaltan la importancia de dónde y cuándo ocurre el crimen, cuyas características físicas y sociales constituyen el objetivo de la misma (Guillén, 2013). En este sentido, los precursores de este nuevo enfoque de analizar la criminología provienen de la Estadística Moral del siglo XIX, caracterizada por ser pionera en la producción de mapas del delito y por correlacionar las condiciones sociales con los delitos; autores como Quetelet y Guerry (Beirne, 1993), permitieron demostrar la existencia de patrones geográficos en la distribución del delito así como su consolidación en el tiempo (Sanz \& Guillén, 2011); y la Escuela de Chicago que destacó por la hipótesis de que los individuos marginales socialmente tienden a concentrarse en un espacio particular de características similares, generando un área cultural aislada (Wortley \& Mazerolle, 2013). Por otro lado, existen puntos de confluencia entre la Criminología Ambiental y la Geografía Social, en tanto que ésta última relaciona la sociedad de un determinado territorio con su espacio geográfico, teniendo como objetivo la interacción de los factores geográficos con una población (Pillet Capdepón, 2004).

La conducta infractora de la población ha sido objeto de análisis en múltiples investigaciones (Morales, Molina, Struse, \& Belmonte, 2014; Sanz \& Guillén, 2011; Vozmediano \& San Juan, 2011; Guillén $\&$ Sanz, 2006) relacionadas con la Criminología Ambiental y las oportunidades delictivas. Así pues, los denominados "Hot Spots" suponen un importante avance en la localización espacial del delito. Estos Hot Spots o Puntos calientes representan una zona concreta donde tiene lugar una elevada concentración de incidentes delictivos en una determinada región (González \& Urpina, 2013), de tal manera que constituyen lugares pequeños donde en un periodo mínimo de un año se puede predecir el delito debido a su incidencia (Sherman, 1995).

A través de las herramientas SIG [Sistemas de Información Geográfica] se posibilita la visualización de los mencionados puntos calientes. Los mapas resultantes (mapas del crimen o del delito) se definen como el proceso de utilización de un SIG para llevar a cabo un análisis especial de los problemas de la delincuencia, realizando un análisis visual y estadístico de la criminalidad y otros eventos que facilitan la vinculación de los datos sobre la delincuencia con otras fuentes de datos, lo que permite establecer relaciones entre las variables (Santos, 2012). En definitiva, proporciona mapas para explicar visualmente los resultados del análisis de estudio (Bachman \& Schut, 2008). Por tanto, la delincuencia tiene un innegable componente geográfico (Clavell \& Oliveras, 2011).

Tras la revisión de la literatura científica, en cualquier caso, no se han analizado los escenarios y los patrones espaciales y temporales de los incidentes de los barrios de la ciudad de Murcia, únicamente existe un análisis urbanístico de barrios vulnerables realizado por el Ministerio de Fomento para el año 2006. Por tanto, esta tarea podría abordarse tanto desde la perspectiva de la Criminología Ambiental como de la Geografía Social. Así pues, con la realización de este trabajo lo que se pretende es adquirir una mayor comprensión del fenómeno delictivo desde estos dos puntos de vista, atendiendo especialmente a las diferentes teorías en este campo y sobre todo al uso de Sistemas de Información Geográfica, que nos pueden hacer ver cómo las características del ambiente pueden generar más oportunidades delictivas en determinados contextos. Todo ello con el fin de contribuir a la prevención de la conducta infractora en la ciudad de Murcia.

\subsection{Objetivos e hipótesis}

El objeto principal de este trabajo es explicar la utilidad de los SIG [Sistemas de Información Geográfica] para la Criminología y la Geografía Social, especialmente en la elaboración de mapas del delito que permiten concretar visualmente el fenómeno delictivo de una determinada zona. Concretamente, los objetivos de este trabajo son: conocer las características principales de los barrios de la ciudad de Murcia, dividiendo éstos en zonas, para detectar e identificar aquellas vulnerables donde las oportunidades delictivas son más altas debido en principio a factores socioeconómicos como el nivel de desempleo, de estudios, o las carencias en las viviendas o infraestructuras; analizar y recolectar los incidentes de segu- 
ridad ciudadana desde el año 2014 para posteriormente elaborar mapas del delito y analizar los índices de densidad del fenómeno delictivo para determinar los "Hot spots" en el área de estudio empleando la tecnología SIG; analizar a su vez, la relación entre la ubicación geográfica de ciertas categorías delictivas y espacios que han mostrado una especial vulnerabilidad social; y por último, proponer algunas medidas de actuación a través de herramientas SIG.

Por otro lado, se formulan las siguientes hipótesis: el delito de los barrios con elevado número de extranjeros y heterogeneidad cultural será mucho mayor que en aquellas nuevas zonas residenciales de la periferia; el delito se concentrará en mayor medida por la noche en aquellos espacios de ocio, y por tanto, con aglomeraciones; en última instancia, en los barrios más desfavorecidos o vulnerables tendrá lugar una mayor delincuencia.

\subsection{Rasgos del área de estudio}

El área de estudio (Figura 1) comprende los barrios de la ciudad de Murcia, perteneciente al municipio de Murcia, que constituye el principal núcleo urbano de la Región, junto a Cartagena. El casco urbano de Murcia es una entidad singular que integra a 28 núcleos de población o barrios $\left(13 \mathrm{~km}^{2}\right)$, divididos por el río Segura, y casi todos circunvalados por Autovías (A-30) y Rondas (Ronda Norte y Sur) y que se caracteriza por su dinámica poblacional. En la tabla 1, se muestran el número de habitantes de la aglomeración urbana para el año 2015, el cual ha experimentado un vacío poblacional a lo largo de los últimos 10 años.

Figura 1. Área objeto de estudio

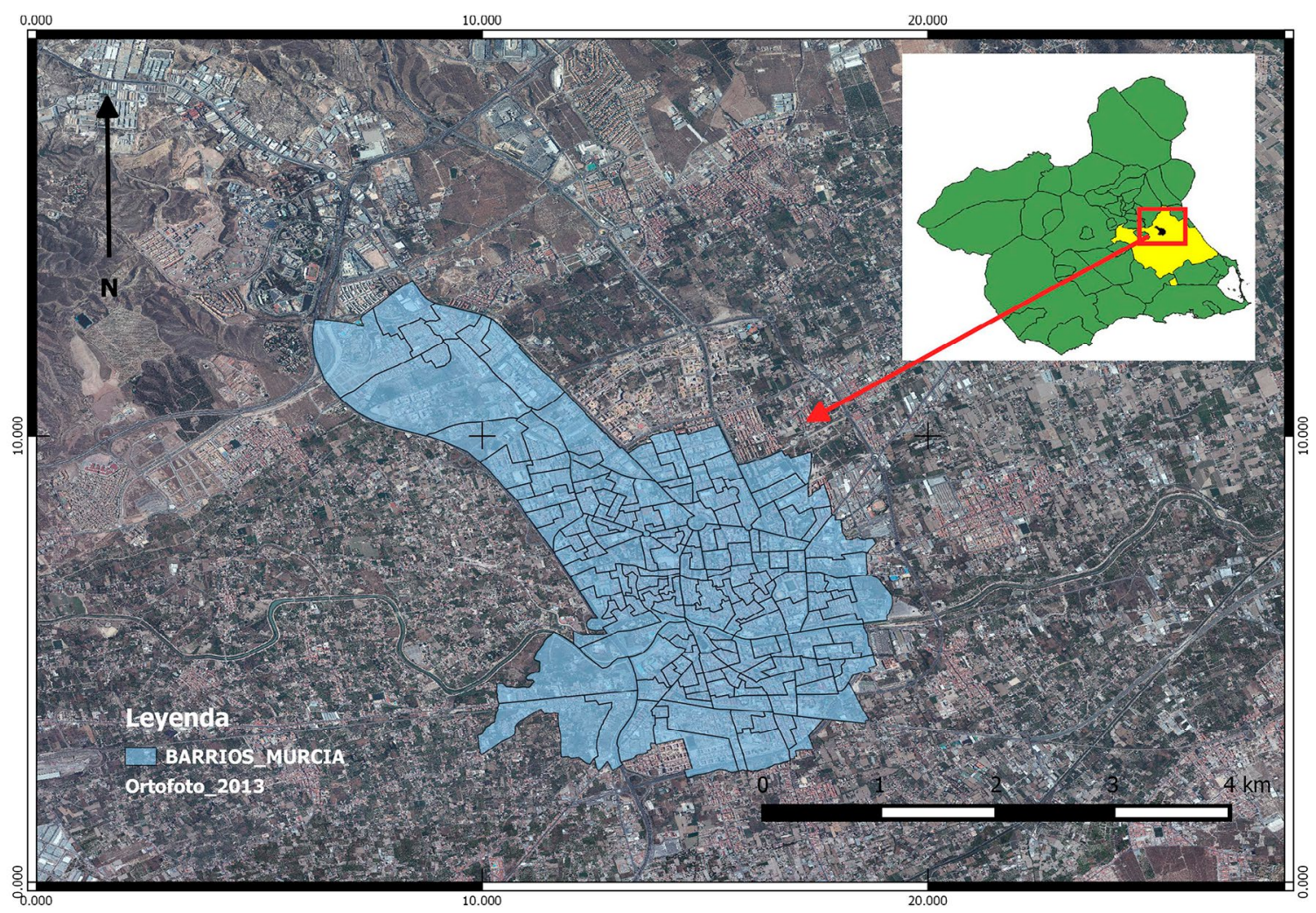

Fuente: Instituto Geográfico Nacional [IGN] y Cartografía de la Región de Murcia [Cartomur]. Elaboración propia.

La ciudad de Murcia se ha ido convirtiendo en un lugar central definido por la terciarización de su economía, la innovación y la cultura. Esta circunstancia ha originado un desmesurado proceso de urbanización donde la gestión del medio ambiente y la calidad de vida constituyen sus retos fundamentales. Retos en los que la movilidad urbana, impuesta por la nueva dinámica, juega un papel decisorio. Esta profunda transformación conduce a la ciudad a ejercer una nueva funcionalidad en el ámbito territorial de su dominación. Una funcionalidad que debe ser interpretada a la luz de la nueva concepción con que se examinan los factores funcionales que definen la vida cotidiana de la ciudad (Andrés Sarasa, 1999). 
En el año 2015, la población total de la ciudad de Murcia era de 168.925 habitantes, según el padrón municipal de habitantes elaborado por el CREM [Centro Regional de Estadística de Murcia]. Esa cifra supone un gran descenso en comparación con la del año 2010, que se situaba en los 178.432 habitantes.

Por otro lado, la población total de extranjeros era de 21.106 habitantes, según el padrón municipal de habitantes (CREM, 2016). En esta cifra también se observa un gran descenso en comparación con la del año 2010, que se situaba en los 27.726 habitantes.

Tabla 1. Número de habitantes y extranjeros por barrios (año 2015)

\begin{tabular}{|c|c|c|c|}
\hline BARRIOS & $\mathrm{N}^{\circ} \mathrm{HAB}$ & $\mathrm{N}^{\circ}$ EXTRANJEROS & EXT/HAB (\%) \\
\hline Buenos Aires & 2787 & 775 & 27,80 \\
\hline Carmen (El) & 20215 & 4133 & 20,44 \\
\hline Catedral (La) & 2211 & 135 & 6,10 \\
\hline Espinardo & 11423 & 840 & 7,35 \\
\hline Fama (La) & 6639 & 646 & 9,73 \\
\hline Flota (La) & 8135 & 416 & 5,11 \\
\hline Infante Juan Manuel & 13226 & 928 & 7,01 \\
\hline Nuestra Sña La Fuensanta & 395 & 28 & 7,08 \\
\hline $\mathrm{Paz}(\mathrm{La})$ & 4774 & 1241 & 25,99 \\
\hline Purísima (La)-Barriomar & 3985 & 1295 & 32,49 \\
\hline Ranero (El) & 4658 & 374 & 8,02 \\
\hline San Andrés & 2243 & 500 & 22,29 \\
\hline San Antolín & 5179 & 1341 & 25,89 \\
\hline San Antón & 8340 & 745 & 8,93 \\
\hline San Bartolomé & 1165 & 89 & 7,63 \\
\hline San Basilio & 5673 & 611 & 10,77 \\
\hline San Juan & 4912 & 909 & 18,50 \\
\hline San Lorenzo & 3027 & 314 & 10,37 \\
\hline San Miguel & 7586 & 461 & 6,07 \\
\hline San Nicolás & 3260 & 627 & 19,23 \\
\hline San Pedro & 1804 & 298 & 16,51 \\
\hline San Pío X & 3050 & 748 & 24,52 \\
\hline Santa Catalina & 1567 & 111 & 7,08 \\
\hline Santa Eulalia & 3001 & 627 & 20,89 \\
\hline Santa María de Gracia & 12512 & 1156 & 9,23 \\
\hline Santiago El Mayor & 9069 & 1412 & 15,56 \\
\hline Vista Alegre & 14250 & 1618 & 11,35 \\
\hline Vistabella & 3839 & 625 & 16,28 \\
\hline
\end{tabular}

Respecto a la población por barrios, por orden de volumen poblacional, aparecen El Carmen seguido del Infante Don Juan Manuel, Vista Alegre, Espinardo y Santa María de Gracia. Estos barrios a su vez coincidirán, como se verá más adelante, con aquellas zonas donde se concentra mayoritariamente el fenómeno delictivo.

Por último, aquellos barrios con mayor población, como es el caso del barrio del Carmen tendrán a su vez, mayores concentraciones de inmigrantes (4133 extranjeros). Y por tanto, una elevada heterogeneidad cultural.

Durante el siglo XIX, la ciudad de Murcia experimentó un gran desarrollo expandiéndose al otro lado del río, constituyéndose el Barrio del Carmen. En el siglo XX se produce la apertura de la Gran Vía de Salzillo y se desarrollaron los ensanches y barrios periféricos, en torno a las salidas hacia Madrid y Barcelona. El crecimiento en polígonos se localiza fundamentalmente al Este de la ciudad (Santa María de Gracia, 
Polígono Infante don Juan Manuel, Vistabella y La Paz). Al Oeste, la trama urbana es confusa, mientras que el crecimiento de la población llevó a asentarse lo largo de la carretera de Madrid hacia Espinardo, donde se produce una combinación en la trama de las antiguas huertas con los nuevos polígonos (Andrés Sarasa, 1999).

Como en la mayoría de las ciudades españolas, sus respectivos centros históricos han experimentado un cierto vacío en un momento determinado. El centro del municipio de Murcia sufrió toda una sangría emigratoria desde 1950 hasta 1975, de forma que ese vacío, desde el punto de vista demográfico, repercutió en la dinámica poblacional del casco urbano de Murcia, de tal manera que actualmente aún son visibles las consecuencias de aquellos años de vacío poblacional, con elevadas tasas de población envejecida y por el contrario, bajas tasas de población joven que únicamente ven en la ciudad un lugar donde trabajar, residiendo en otros espacios situados a las afueras del centro (Martí Ciriquián, \& Moreno Vicente, 2014). A su vez, el vacío demográfico de la ciudad de Murcia se solucionará, en parte, por la inmigración, lo que ha ocasionado unos elevados índices de extranjeros repartidos por los distintos barrios de la ciudad. Estos inmigrantes procederán tanto de América, Europa del Este, así como de África por su mayor proximidad. Por barrios ocupan con porcentajes destacables sobre el número de habitantes total, Barriomar $(32,49 \%)$, Buenos Aires $(27,80 \%)$ y La Paz $(25,99 \%)$ mientras que El Carmen tiene el mayor número de inmigrantes, aunque su importancia respecto al resto de habitantes del mismo barrio no es tan alta como en otras zonas (www.carm.es).

Asimismo, durante los años 60,70 y 80 se produce todo un proceso urbanizador por parte de la administración caracterizado por edificios de escasa calidad, espacio reducido, etc., ubicándose en dichas zonas grupos de personas de similares características, principalmente etnia gitana, con escasos recursos... Por lo tanto, el origen de barrios más desfavorecidos en la ciudad de Murcia va unido al desarrollo urbanístico, con la creación de nuevas zonas residenciales como La Paz, La Fama, Espíritu Santo etc., motivado tanto por el crecimiento de la natalidad durante el denominado "Baby Boom" de los años 60, así como por la masiva llegada de población inmigrante desde finales de los años 80, por ejemplo, en San Andrés, Barriomar, La Paz y Buenos Aires, procedentes en primer lugar del Magreb y posteriormente del África Subsahariana, Europa del Este y Latinoamérica (Serrano Rodríguez \& Martínez Valero, 2013).

De esta manera, socialmente ha habido todo un cambio, pues los habitantes que residían en la ciudad se han unido un elevado número de inmigrantes, de forma que ha conllevado ciertas repercusiones negativas como el racismo, la xenofobia, etc. Como sostiene Sassen (2008): "Las ciudades son el marco en el que personas de muchos países diferentes tienen más probabilidades de coincidir y lo mismo ocurre con una multiplicidad de culturas". (p.42)

El paisaje urbano de la ciudad de Murcia quedará fragmentado, con características muy diversas en función del tipo de construcciones, número de habitantes, grupos sociales etc. Es por tanto, que el comportamiento de los habitantes de los barrios de Murcia se adapta de forma condicionada al espacio que habitan (Serrano Rodríguez \& Martínez Valero, 2013). Además, en la ciudad surgen áreas donde determinados grupos sociales con diferencias significativas encuentran las condiciones más adecuadas para su "estilo de vida" y a los que irremediablemente acuden. Como argumenta Fisher (2011), "Las personas eligen donde vivir en función de sus condiciones socioeconómicas pero también dependiendo de sus redes sociales, grupos de pertenencia y de sus deseos". (p.192)

\subsubsection{Concepto de barrios desfavorecidos}

Las zonas desfavorecidas responderán, por lo general, a una situación compleja donde uno de los aspectos más importantes a tener en cuenta es la carencia de recursos que impide a la población a disfrutar de una calidad de vida en igualdad con otros sectores o zonas de la ciudad (Arias, 2000). A ello hay que añadir que una zona o barrio desfavorecido se caracterizará por una debilidad estructural desde el punto de vista social y demográfico, así como en sus cualidades ambientales del espacio urbano que lo diferencian del resto de la ciudad. No obstante, como señala Hernández Ajá (1996) hay que diferenciar el concepto de desfavorable del concepto de vulnerable, siendo éste último una situación mucho más crítica, de forma que de no actuar sobre el problema, la zona entraría en crisis pudiendo producirse una degradación social y funcional. Mientras que en barrios desfavorecidos se delimita el espacio, con carencias materiales que podrían solventarse a partir de medidas de planificación y legislación. Para ser considerado un barrio desfavorecido y obtener subvención mediante proyectos europeos como Urban, como es el caso del 
barrio de Espíritu Santo o el de Barriomar, se deben reunir, al menos, 3 de los siguientes requisitos: bajo nivel educativo, baja actividad económica, alta tasa de paro, elevada delincuencia, degradación ambiental y tendencia demográfica desfavorable (Arias, 2000).

\subsubsection{Zonas vulnerables}

Según Fisher (2011) se pueden señalar fundamentalmente cuatro zonas vulnerables en la ciudad de Murcia, que compartirán diferentes características, entre las que sobresalen una elevada concentración de viviendas sociales y una presencia significativa de población gitana e inmigrante:

- Centro de la ciudad: caracterizada por una alta tasa de población envejecida, demografía desfavorable, pocos inmigrantes (no alcanza los 500 extranjeros entre los distintos barrios del centro de la ciudad), altos niveles educativos, alta urbanización con precios elevados de viviendas.

- Zonas urbanas colindantes al centro (San Andrés, El Carmen, Buenos Aires): según el CREM (2016), cuenta con una población envejecida (hay 5769 personas con más de 65 años) y una importante presencia de inmigrantes (5408 extranjeros entre estos barrios, siendo El Carmen el barrio con más inmigrantes) dando una imagen negativa del área por una disminución de población de origen autóctono. El nivel de estudios también es llamativo, pues solo en El Carmen hay más de 2827 personas sin estudios (CREM, 2016).

- Zonas de urbanización reciente: incluye zonas del norte y sur de la ciudad con una ausencia de inmigrantes, tendencia demográfica favorable, constituyendo una zona básicamente residencial que no requieren atenciones sociales significativas.

- Zonas periféricas con precariedad social (La Fama, Barriomar, La Paz, Espíritu Santo, Infante): altos índices de delincuencia, drogadicción y violencia, con imágenes negativas desde la perspectiva social. Cuenta con una elevada presencia de inmigrantes (más de 3500 extranjeros concentrados en su mayoría en La Paz y Barriomar) y bajos niveles educativos (en La Fama y Barriomar cerca de 1000 personas no tienen estudios, en La Paz 1371 y en el Infante 1446 personas). Algunos de estos barrios como La Fama surgieron como barrios de lujo, pero el Ayuntamiento favoreció la construcción de viviendas sociales, especialmente en la c/ Santa Rita, que actualmente es el mayor foco de vente de drogas al por menor de Murcia.

\section{Metodología}

Es un estudio regional, en el marco del uso de nuevas tecnologías para representar, analizar y prevenir el fenómeno delictivo, siguiendo el modo de trabajo de autores como (Fernández Molina, Vázquez, \& Belmonte, 2013; Guillén \& Sanz, 2006; Vozmediano \& San Juan, 2006; González \& Urpina, 2013). El análisis se ha basado en el estudio de todos los datos de los incidentes de seguridad ciudadana desde el año 2014 hasta la actualidad a través de fuentes publicadas por la policía local de Murcia en redes sociales como "Twitter" o en la página web del ayuntamiento de Murcia, actualizadas día tras día con los sucesos más relevantes. Se ha realizado una muestra de 5.771 publicaciones, contabilizándose solamente aquellas que tenían relación con el espacio objeto de estudio así como con los años analizados, resultando un total de 155 incidentes válidos.

La búsqueda de datos no ha sido del todo fructífera ya que ningún organismo público ofrecía datos de manera individualizada, por tanto, visitas a la delegación del gobierno, policía o fiscalía no pudieron aportar datos de interés para el estudio y relevantes para la investigación, teniendo que delimitar el estudio a otras fuentes como las mencionadas con anterioridad y que en un principio pueden no parecer muy fiables pero demuestran ser de gran utilidad para ver de forma intuitiva la criminalidad en Murcia.

En este sentido, la validez y fiabilidad de las distintas fuentes de información sobre la criminalidad no es completa, debido precisamente a la multitud de fuentes, muchas veces contradictorias entre sí. Otro gran problema a tener en cuenta es el de la cifra negra, relacionado con el iceberg de la delincuencia, donde la mayor parte del fenómeno criminal sería delincuencia oculta ya que en la mayoría de los casos ni siquiera existe una denuncia, por lo que no queda registrado.

Para finalizar, ha sido de gran utilidad los archivos con formato .shp para la representación cartográfica obtenidos en el Instituto Geográfico Nacional [IGN] y Cartomur, así como el uso del programa QGIS (software libre) para la elaboración de mapas. 
Por su utilidad para ubicar la distribución del delito, se ha utilizado mapas con información referente a:

- Zonas verdes, plazas y jardines

- Centros comerciales, tiendas, comercios, restaurantes, bancos, aparcamientos, etc.

- Calles y carreteras

- Centros sanitarios y escolares

- Instalaciones culturales y deportivas

- Comisarías policiales y bomberos

- Lugares de culto, etc

Mientras que sobre el resto de capas se ha incluido una capa semitransparente que muestra la división de barrios individualmente y agrupados por zona.

\section{Resultados}

Para el análisis del fenómeno delictivo se propone relacionar el balance de criminalidad elaborado por el Ministerio del Interior en todo el municipio de Murcia con el número de delitos que tuvieron lugar en el mismo periodo de tiempo en los barrios de la ciudad de Murcia.

\subsection{Balance de criminalidad en el municipio de Murcia}

El aumento del acto delictivo en el municipio de Murcia en los años objeto de estudio es un hecho palpable en distintos medios de comunicación como: La Opinión (2016), Diario La Razón (Ussía Alfonso, 2012), Diario 20 minutos (2015) etc., que tienen como punto de referencia el balance de criminalidad que trimestralmente se publica en el Ministerio de Interior.

Tabla 2. Balance de criminalidad del municipio de Murcia (2014-2015)

\begin{tabular}{|l|r|r|r|}
\hline \multicolumn{1}{|c|}{ TIPOLOGÍA PENAL } & \multicolumn{1}{|c|}{2014} & 2015 & Var. \% 15/14 \\
\hline 1.-HOMICIDIOS DOLOSOS Y ASESINATOS (EU) & 0 & 1 & 100 \\
\hline 2.-ROBO CON VIOLENCIA E INTIMIDACIÓN (EU) & 637 & 678 & 6.4 \\
\hline 3.-ROBOS CON FUERZA EN DOMICILIOS (EU) & 1.450 & 1.467 & 1,2 \\
\hline 4.-SUSTRACCIÓN VEHÍCULOS A MOTOR (EU) & 584 & 657 & 12,5 \\
\hline 5.-TRÁFICO DE DROGAS (EU) & 62 & 68 & 9,7 \\
\hline 6.-DAÑOS & 1.296 & 1.360 & 4,9 \\
\hline 7.-HURTOS & 5.823 & 6.251 & 7,4 \\
\hline
\end{tabular}

Fuente: Ministerio del Interior (2016) (http://www.interior.gob.es/prensa/balances-e-informes/2015). Elaboración propia.

Entre los resultados más llamativos de la tabla 2 , destaca el aumento considerable de hurtos $(7,4 \%)$ y sustracción de vehículos a motor $(12,5 \%)$, siendo poco representativa la variación de homicidios (de 0 a 1 entre 2014 y 2015). El balance recoge datos de Policía Nacional, Guardia Civil, Ertzaintza, Mossos d'Esquadra y Policía Foral de Navarra, así como de cuerpos de Policía Local que facilitan datos al Sistema Estadístico de Criminalidad. Además, debido a la entrada en vigor del nuevo Código Penal, Interior y las Fuerzas y Cuerpos de Seguridad están adaptando el Sistema Estadístico de Criminalidad (SEC) para la correcta recopilación y registro de las nuevas tipologías penales. El conjunto de datos a nivel municipal supondrán la base para el análisis más exhaustivo de información espacial en la ciudad de Murcia, con datos procedentes del Ayuntamiento de Murcia (2016) y de la Policía Local de Murcia (2016) que publica en sus redes sociales.

\subsection{Mapas del delito de la ciudad de Murcia}

La localización de los diferentes delitos acaecidos en los últimos 3 años se reparte en los 28 barrios que constituyen la ciudad de Murcia. Se puede apreciar una mayor concentración del delito en la parte Norte (especialmente la barriada Espíritu Santo de Espinardo), en la parte central (concretamente La Fama) y en el Sur (El Carmen), constituyendo éste último el barrio que más incidentes delictivos registró desde 2014 hasta la actualidad (Figura 2). 
Figura 2. Distribución del número de delitos en la ciudad de Murcia (enero 2014-junio 2016)

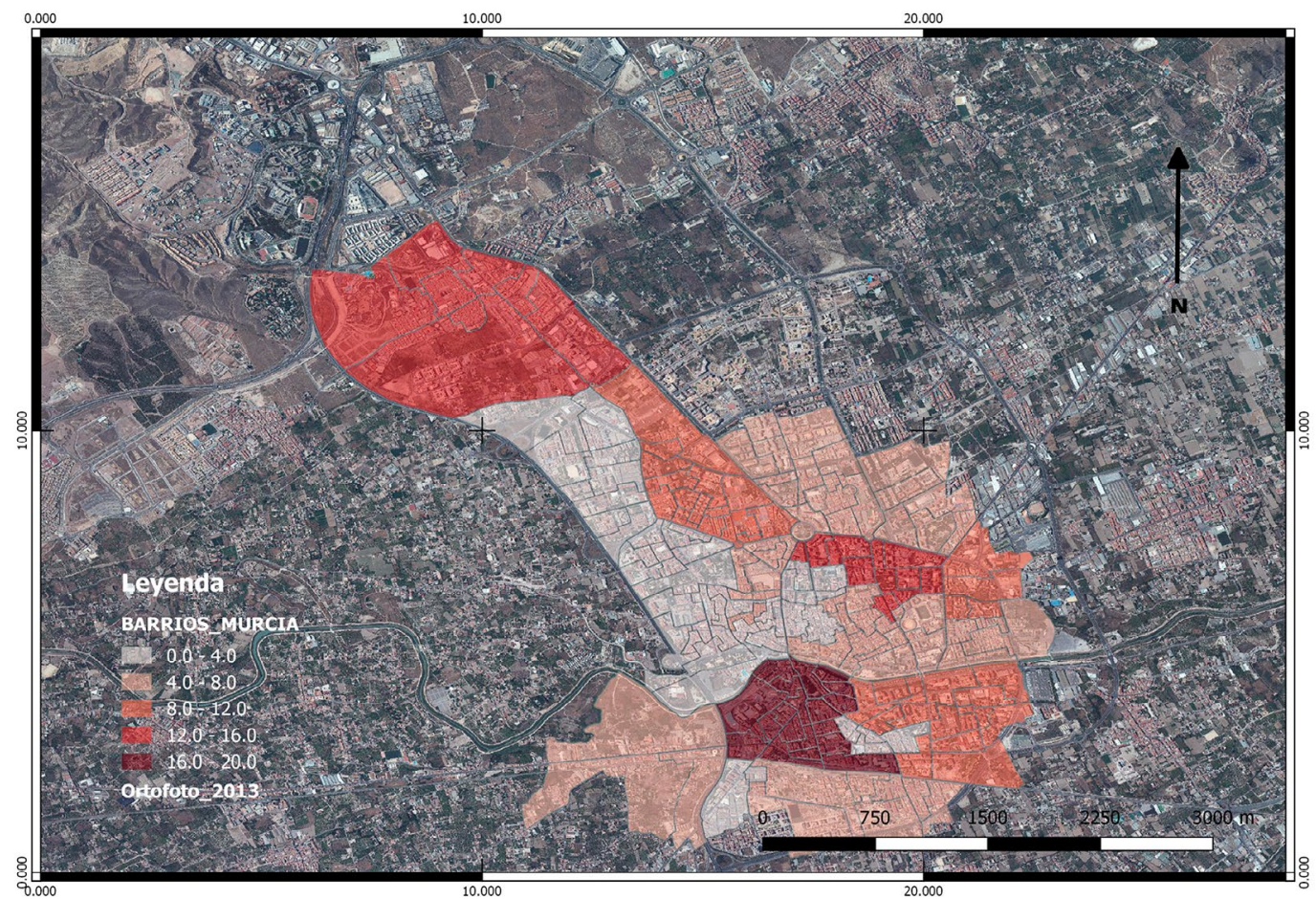

Leyenda: $\mathrm{N}^{\mathrm{o}}$ de delitos: 0-4; 4-8; 8-12; 12-16 y 16-20.

Fuente: IGN, Cartomur y Ayuntamiento de Murcia. Elaboración propia.

Figura 3. Zonas vulnerables y especialmente conflictivas de la ciudad de Murcia

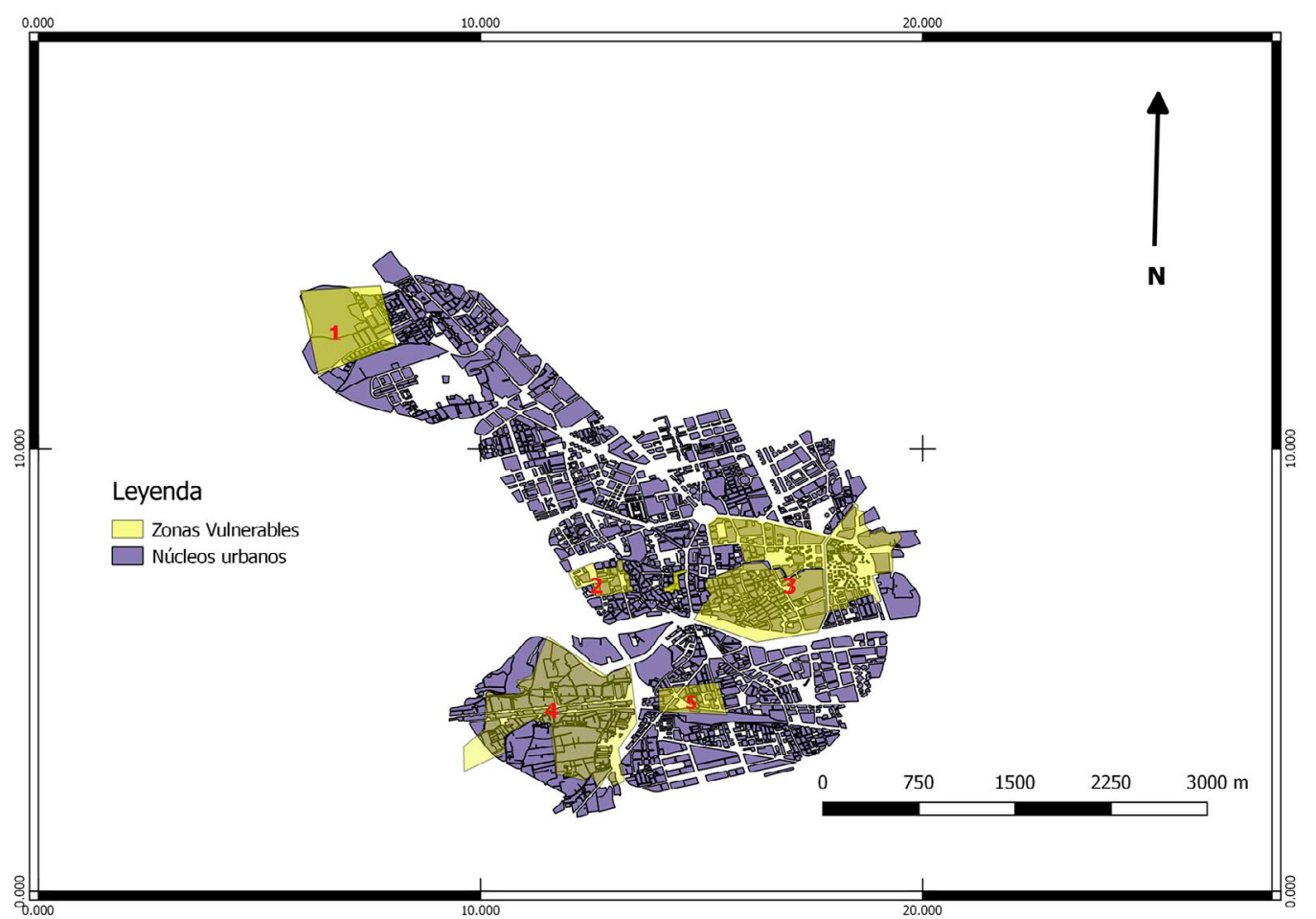

Etiquetado: 1-Espiritu Santo; 2-San Andrés; 3- La Paz, La Fama y el casco (San Bartolomé, San Juan y Santa Eulalia) 4-Barriomar y 5-El Carmen (Estación).

Fuente: IGN, Cartomur y Ayuntamiento de Murcia. Elaboración propia. 
Figura 4. Ubicación geográfica de los distintos delitos cometidos en la ciudad de Murcia

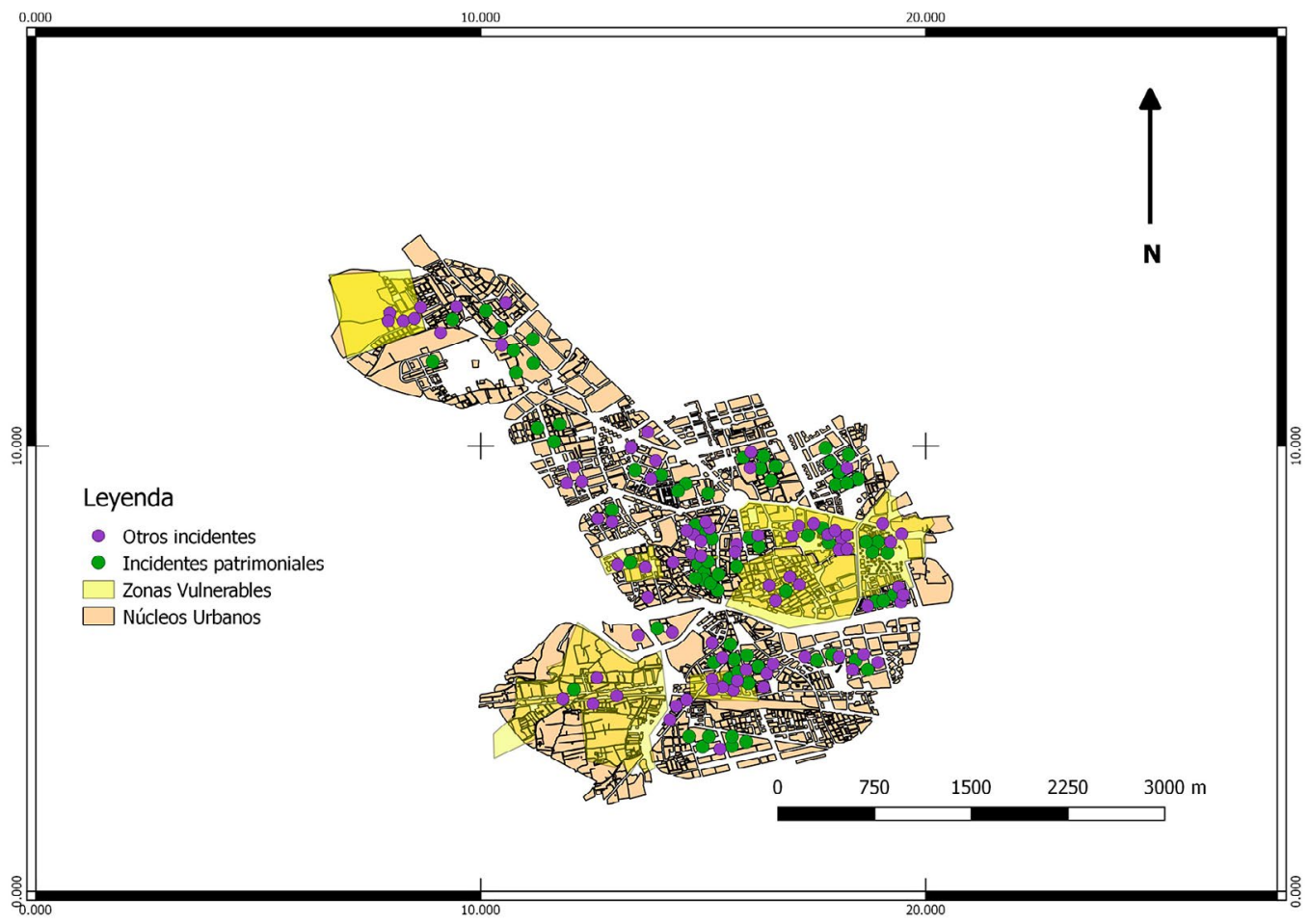

Fuente: IGN, Cartomur y Ayuntamiento de Murcia. Elaboración propia.

Figura 5. Distribución temporal (día y noche) de los delitos analizados en la ciudad de Murcia

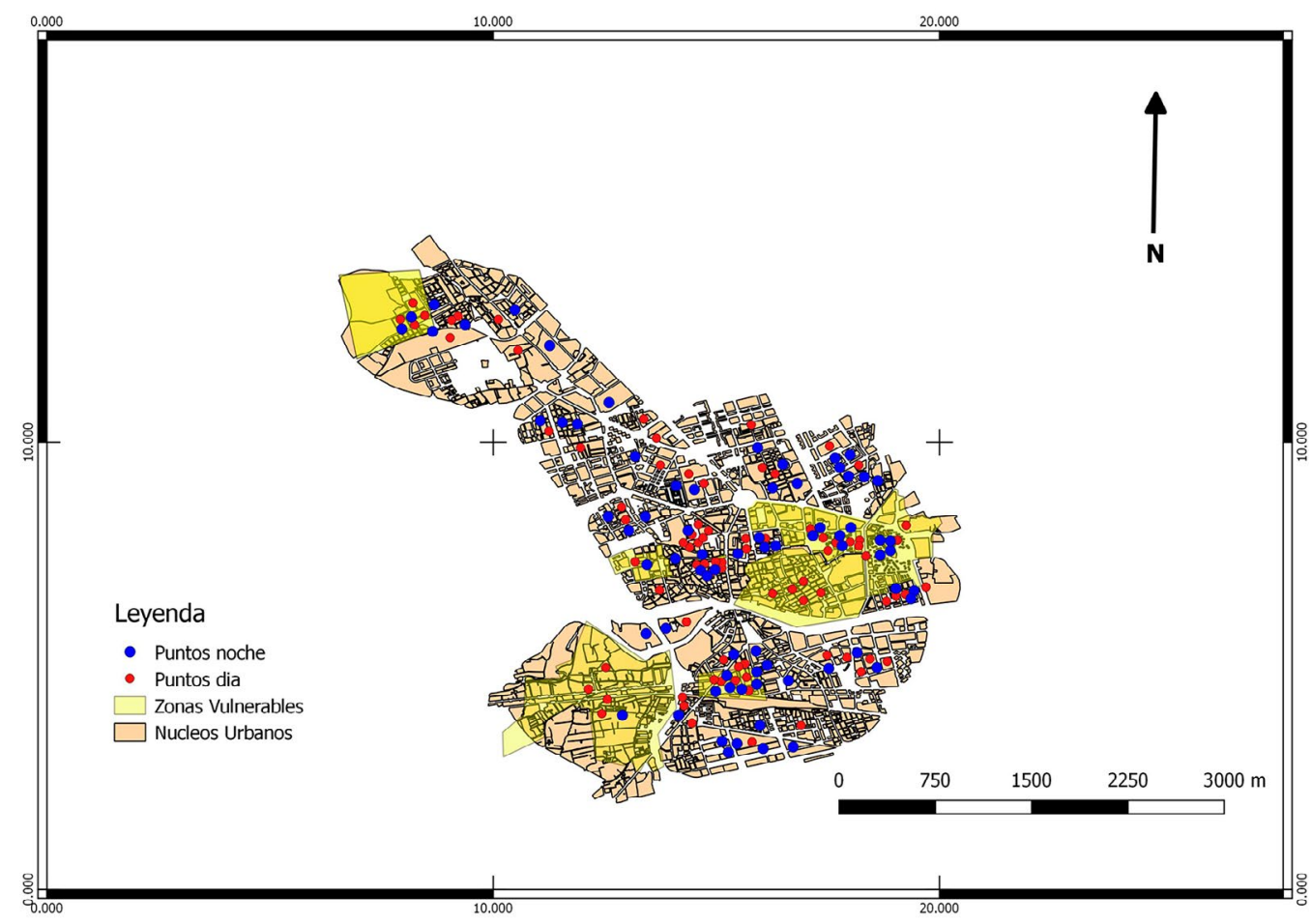

Fuente: IGN, Cartomur y Ayuntamiento de Murcia. Elaboración propia.

Por lo general, si se atiende a la naturaleza de los delitos analizados, se puede considerar una tendencia a los delitos contra el patrimonio (hurto, robo con fuerza, con intimidación, y con vehículo a motor) 
que configuran las conductas más frecuentes. Una vez definidos los principales barrios donde se da un mayor número de delitos, interesa analizar el patrón que sigue estos incidentes respecto a las zonas vulnerables de la ciudad, que aparecerán sombreadas de color amarillo.

Según Hernández Ajá et al., 2006 y observando la figura 3, se puede comprobar que existen 5 zonas vulnerables en la ciudad de Murcia donde, por el contrario, no siempre tiene lugar un gran número de delitos (caso de San Andrés y el casco antiguo). Esta distribución de zonas podría explicarse atendiendo al Modelo Concéntrico de Burguess (1925) por el que las ciudades se desarrollan a partir de un núcleo central donde se ubican los principales comercios y centros de negocios, creciendo en forma concéntrica o anillos caracterizados cada uno de ellos por una población y unas actividades socioeconómicas bien diferenciadas (Fernández Molina et al., 2013). Así pues, aquellos espacios residenciales de reciente construcción registran un número de delitos mucho menor, pues si los resultados se apoyan en que el urbanismo influye en la delincuencia, aquellos barrios de transición entre el centro histórico y los nuevos espacios urbanos, caracterizados por una heterogeneidad cultural y degradación de la vivienda, transmiten una mayor actividad delincuencial (Sherman, 1995). No obstante, y como sostienen Fernández Molina et al., (2013) no deben realizarse afirmaciones deterministas al respecto sobre esta relación del urbanismo con el número de delitos, pues también puede verse afectado por un mayor o menor volumen poblacional de esas zonas.

Como se ha comentado anteriormente, el análisis se centra fundamentalmente en delitos patrimoniales que son los que más sobresalen en la ciudad de Murcia en el periodo de tiempo analizado, de forma que se ha categorizado los incidentes delictivos entre patrimoniales $u$ otros incidentes. Como se puede observar en la Figura 4, los hot spots de los delitos se concentran claramente en 3 zonas de la ciudad. Al Norte, aparece un primer patrón espacial delictivo entorno a los barrios periféricos de Espinardo (Espíritu Santo), coincidiendo prácticamente con la primera zona vulnerable descrita, con bajos niveles educativos, viviendas antiquísimas convertidas en chabolas,... aunque en los últimos años el barrio ha sufrido una remodelación integral. En la parte central, aparece una tendencia delictiva en el casco antiguo y sus proximidades (Gran Vía, Campus La Merced, La Fama, etc.), caracterizada por las aglomeraciones urbanas, actividades comerciales y de ocio (donde se concentran la mayor parte de los pubs y bares de la ciudad) que suponen un factor idóneo para los delitos de tipo patrimonial. Mientras que por otro lado, en la parte más meridional de la ciudad de Murcia, correspondiente a los barrios del Infante y El Carmen, se da otro patrón delictivo como consecuencia de sus elevados índice de población, grandes concentraciones de inmigrantes entorno a la estación de trenes del Carmen y la baja calidad urbana de la zona.

A su vez, las zonas vulnerables no siempre coinciden con los puntos donde se concentran el mayor número de incidentes, de manera que los hot spots se encuentran próximos a las zonas vulnerables, pero en todo caso fuera o en sus límites. Entre los aspectos que pueden tener relación con este fenómeno destaca el desplazamiento de los delincuentes en barrios marginales con la finalidad de actuar en otras zonas donde no puedan ser reconocidos y por la mayor concentración y presión policial que presenta estos barrios vulnerables.

En última instancia, para la elaboración de la Figura 5 se ha empleado la variable "Hora", referente al momento del día en el que se ha producido el delito. De esta forma, el mapa queda categorizado en puntos de día (si el delito tuvo lugar entre las 8:00 horas y las 20:00 horas) y puntos de noche (si el delito tuvo lugar entre las 20:00 horas y las 8:00 horas). Entre los principales resultados a tener en cuenta, destaca un mayor número de hechos delictivos de noche en aquellas zonas de ocio (por ejemplo: la zona de las Tascas y Atalayas), aunque por lo general, los puntos temporales se distribuyen de forma heterogénea en el territorio.

\section{Discusión de resultados}

En la Región de Murcia el estudio del fenómeno delictivo a partir de herramientas SIG es escaso o prácticamente nulo. La aplicación de esta técnica en el campo de la Criminología Ambiental empieza a estar en auge en España, como muestra los trabajos de Fernández Molina et al., (2013) sobre la ciudad de Albacete, siendo recientemente cuando se representó la distribución del patrón delictivo sobre dicha ciudad.

El presente trabajo llega a la misma conclusión que el anteriormente citado: en las zonas residenciales con viviendas de reciente construcción, el delito es muy inferior al observado en las zonas de transición, 
entre el centro de la ciudad y las áreas periféricas, donde tiene lugar la mayor concentración de personas con bajo nivel adquisitivo y con viviendas deterioradas y/o de antigua construcción.

En el caso europeo, aparecen trabajos aplicados en la ciudad de Vilna (Lituania) para el año 2014, donde se diseñan un conjunto de mapas, representando características específicas de los delitos, así como la densidad y distribución temporal de crímenes en torno a esta ciudad. También muestran los cambios

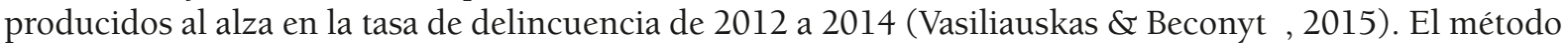
de trabajo es similar al llevado a cabo para este caso de estudio, con la salvedad de que se incorporan visualizaciones en 3D y los mapas fueron generados con datos mucho más detallados y oficiales.

Más exhaustivos son los trabajos realizados sobre EEUU, como es el caso de Albany (capital del estado de Nueva York), que tiene unas características diferentes al de otros estudios previos de la distribución del delito localizados en Seattle y Vancouver (Wheeler, Worden \& McLean, 2015). En estas ciudades se emplea una metodología mucho más compleja con métodos estadísticos que logren establecer los movimientos y trayectorias de los grupos criminales sobre el espacio. Caso de estudio muy parecido es el llevado a cabo por De Melo, Matías \& Andresen (2015) sobre la ciudad brasileña de Campinas ubicada en el estado de Sao Paulo. Ambos trabajos comparten un objetivo común al presentado en este artículo: concretar visualmente el comportamiento del fenómeno delictivo de una ciudad.

En definitiva, y comparando los resultados con los de otros autores en distintas ciudades del mundo, se observan factores semejantes a los expuestos en este trabajo, atribuyendo la mayor concentración del delito en aquellos espacios con una mala calidad urbana, altos índices de población inmigrante y bajos niveles adquisitivos y educativos.

\section{Conclusiones}

Tras el análisis de los resultados obtenidos, se puede evidenciar a partir de los mapas del delito realizados que existe una agrupación de 5 zonas vulnerables, que corresponden con los barrios de Espinardo, El Carmen, La Fama, La Paz, San Andrés, Barriomar, y el casco antiguo, sin embargo, resulta llamativo que no siempre coinciden el mayor número de delitos analizados dentro de estas zonas, como es el caso del barrio de San Andrés y el casco antiguo. Así pues, quedaría rechazada la hipótesis inicial que propugnaba una tendencia de localización del delito en zonas vulnerables, ubicándose en sus límites o a las afueras por la mayor presión policial de estas zonas. Por otro lado, la distribución espacial de los delitos se hace especialmente intensa en el barrio de El Carmen como espacio que sobresale en el conjunto global de la ciudad de Murcia, seguido de los barrios de Espinardo y La Fama, si bien hay que tener en cuenta el volumen poblacional de cada zona así como sus condiciones de urbanismo y socioeconómicas para explicar esta situación.

Como se ha argumentado, los resultados espaciales han tenido una mayor significancia que los temporales, ya que éstos últimos, a excepción de zonas de ocio nocturno con elevada presencia de bares y pubs donde hay una tendencia a delitos contra el patrimonio, sobre todo en noches de fines de semana. En cambio, los incidentes que han tenido lugar de día, se concentran muy heterogéneamente en el mapa del delito.

Debe de existir una relación de los Hot Spot o puntos calientes que más sobresalen por sus números de delitos respecto a un mayor control policial de estas zonas, dándose un mayor despliegue de cuerpos de policía en barrios con mayor incidencia delictiva. Por tanto, en el ámbito de la seguridad, puede impulsarse el desarrollo de herramientas de georreferenciación (Sistemas de Información Geográfica) para mejorar la gestión y visualización de la información y facilitar, de esta manera, su intercambio entre plataformas policiales. Es por ello que toma especial relevancia la vinculación de la Criminología con la Geografía, tan olvidada en muchos casos a pesar de tener una gran aportación a esta ciencia.

Así pues, los mapas del delito elaborados mediante herramientas SIG constituyen una manera eficaz y efectiva para mejorar las estrategias de gestión de la seguridad ciudadana, como se ha visto en otros casos de estudio aplicados en la ciudad de Albacete por Fernández Molina et al., (2013). Asimismo, una herramienta vanguardista y novedosa sería la utilización de un plugin para predicción policial a través de puntos espacio-temporales mediante programas como gvSIG o R, pues la sociedad demanda de la policía una situación preventiva y pro-activa, de manera que la predicción de los hechos delictivos mediante este tipo de modelos estadísticos permitirá a la policía disponer de una herramienta que mejore la distribución de sus efectivos y el análisis previo a su actuación. 
Otra alternativa a señalar se trata de la página web crimemapping.com (http://www.crimemapping. $\underline{\mathrm{com} / \mathrm{map} / \mathrm{agency} / 265)}$, en el caso de EEUU, que constituye una comunidad que incluye de manera visual cualquier tipo de incidente ocurrido, desde agresiones sexuales, pasando por homicidios o hurtos. En definitiva, cualquier tipo de delito. Los datos se extraen del sistema de registros existentes en cada agencia de policía, participantes a través de un proceso de importación automatizada. Los datos mostrados siempre están actualizados. Este es un ejemplo que podría implantarse en España y ser de gran utilidad por la veracidad y actualidad de sus datos, así como la facilidad de acceso para cualquier ciudadano o persona interesada.

Para finalizar, de cara a estudios posteriores, sería interesante abordar la temática con una muestra más elevada o incluso con datos oficiales de manera desglosada, barrio por barrio, de la policía o fiscalía. Aunque esto último, en el caso de este estudio no ha sido posible debido a su postura reacia a la hora de facilitar cualquier tipo de datos que no se encuentre en un documento oficial.

\section{Agradecimientos}

Para la realización de este trabajo, ha sido útil el consejo de personas que se involucraron de forma altruista (como Esther Fernández Molina, perteneciente al Centro de Investigación en Criminología de la Universidad de Castilla La Mancha, o Ginés Sánchez Martínez, Guardia Civil encargado de atender las llamadas de los ciudadanos). Dar también las gracias a los revisores por sus observaciones que ayudaron a mejorar el contenido del artículo.

\section{Referencias}

Andrés Sarasa, J. L (1999). Movilidad urbana en la ciudad del Siglo XXI. Papeles de Geografía $n^{\circ} 29$. Universidad de Murcia, 29, 15-34.

Arias, F. (coord) (2000): La desigualdad urbana en España. Madrid. Ministerio de Fomento.

Ariza, J. J. M. (2011). Políticas y estrategias de prevención del delito y seguridad ciudadana. Edisofer.

Ayuntamiento de Murcia (2016). Recuperado de: http://www.murcia.es

Bachman, R., \& Schutt, R. K. (2008). Fundamentals of research in criminology and criminal justice. Sage.

Beirne, P. (1993). Inventing Criminology: Essays on the rise of'homo criminalis'. SUNY Press.

Burguess, E.W (1925): The city. University of Chicago Press.

Cartografía de la Región de Murcia [Cartomur], Infraestructuras de Datos Espaciales de Referencia de la Región de Murcia. Recuperado de: http://cartomur.imida.es/visorcartoteca/)

Centro Regional de Estadística de la Región de Murcia [CREM] (2016). Recuperado de: http://econet. carm.es/

Clavell, G. G., \& Oliveras, M. P. (2011). Crisis económica y gestión de la inseguridad ciudadana: los mapas de delincuencia. Revista Catalana de Seguretat Pública, 24, 79-105.

Comunidad Autónoma de la Región de Murcia [CARM]. Recuperado de: www.carm.es

Crime Mapping (2016). Recuperado de: http://www.crimemapping.com/map/agency/265

De Melo, S. N., Matias, L. F., \& Andresen, M. A. (2015). Crime concentrations and similarities in spatial crime patterns in a Brazilian context. Applied Geography, 62, 314-324. http://doi.org/10.1016/j. apgeog.2015.05.012

Europa Press (23 de julio de 2015). Los delitos y faltas aumentan un 0,4\% en la Región de Murcia, el segundo mayor aumento por CCAA. Diario 20 minutos. Recuperado de: http://www.20minutos.es/ noticia/2520478/0/delitos-faltas-aumentan-0-4-region-murcia-segundo-mayor-aumento-por-ccaa/

Fernández Molina, E., Vázquez, D \& Belmonte, M. (2013). Los puntos calientes de la delincuencia. Un análisis de la distribución espacial del fenómeno delictivo en la ciudad de Albacete. IV Jornadas Ibéricas de Infraestructura de Datos Espaciales, 351-363.

Fisher, O (2011): "Segregación étnica y desigualdad social en el municipio de Murcia". (no publicado).

González, C. V., \& Urpina, C. S. (2013). El análisis geográfico del delito y los mapas de la delincuencia. Revista de derecho penal y criminología, (9), 419-448.

Guillén, C. S. J., \& Sanz, L. V. (2006). Empleo de Sistemas de Información Geográfica en el estudio del Miedo al Delito. Revista Española de Investigación Criminológica: REIC, (4), 2. 
Guillén, C. S. J. (2013). Criminología ambiental: un área en expansión. AIS: Ars Iuris Salmanticensis, 1(1), 37-42.

Hernández Ajá, a (1996): Análisis urbanístico de barrios desfavorecidos. Catálogo de áreas vulnerables españolas. Recuperado de: http://polired.upm.es/index.php/ciur/article/viewFile/233/229

Hernández Ajá, A., Vázquez Espí, M., García Madruga, C., Matesanz Parellada, Á., Moreno García, E., Alguacil Gómez, J., \& Camacho Gutierrez, J. (2006). Análisis Urbanístico de Barrios Vulnerables. Madrid: Ministerio de Fomento.

Instituto Geográfico Nacional [IGN] (2016). Recuperado de: http://www.ign.es/ign/main/index.do

La Opinión (9 de febrero de 2016). Los delitos bajan en toda España menos en Murcia. La Opinión de Murcia. Recuperado de: http://www.laopiniondemurcia.es/comunidad/2016/02/09/delitos-bajanespana-salvo-murcia/712609.html

Martí Ciriquián, P., \& Moreno Vicente, E. (2014). La transformación urbana y territorial de la ciudad de Murcia y su entorno (1977-2010). Estudios Geográficos, 75(276), 261-309. http://dx.doi.org/10.3989/ estgeogr.201407

Ministerio de Fomento (2006). Análisis urbanístico de barrios vulnerables. Recuperado de: https://www. fomento.gob.es/MFOM/LANG_CASTELLANO/DIRECCIONES_GENERALES/ARQ_VIVIENDA/ SUELO_Y_POLITICAS/OBSERVATORIO/Analisis_urba_Barrios_Vulnerables/

Ministerio de Interior (2016). Balance de Criminalidad. Recuperado de: http://www.interior.gob.es/ prensa/balances-e-informes/2015

Morales, D. V., Molina, E. F., Struse, S. P., \& Belmonte, M. (2014). "El perfil geográfico de la delincuencia juvenil: Un análisis de las características espaciales asociadas a la movilidad delictiva de los jóvenes". Revista Española de Investigación Criminológica: REIC, (12), 6-37.

Policía Local de Murcia (2016). Recuperado de: http://policia.murcia.es/web/policia-local/noticias

Pillet Capdepón, F. (2004). La geografía y las distintas acepciones del espacio geográfico. Investigaciones geográficas, 34, 141-154. https://doi.org/10.14198/INGEO2004.34.07

Santos, R. B. (2012). Crime analysis with crime mapping. Sage.

Sanz, L. V., \& Guillén, C. S. J. (2011). Criminología Ambiental: ecología del delito y de la seguridad. Editorial UOC.

Sassen, S. (2008). A sociology of globalization. Análisis político, 20(61), 3-27.

Serrano Rodríguez, I., \& Martínez Valero, N. (2013). Zonas desfavorecidas residencialmente en el municipio de Murcia. En Vivienda y exclusión residencial (pp. 191-219). Editum Ediciones de la Universidad de Murcia.

Sherman, L. W., Gartin, P. R., \& Buerger, M. E. (1989). Hot spots of predatory crime: Routine activities and the criminology of place. Criminology, 27(1), 27-56.

Sherman, L. W. (1995). Hot spots of crime and criminal careers of places. Crime and place, 4, 35-52.

Ussía Alfonso (21 de septiembre de 2012). Aumentan las violaciones y agresiones sexuales en Murcia un 43 por ciento según la Fiscalía. Diario La Razón. Recuperado de: http://www.larazon.es/historico/1325aumentan-las-violaciones-y-agresiones-sexuales-en-murcia-un-43-por-ciento-segun-la-fiscaliaHLLA_RAZON_488458

Vasiliauskas, D., \& Beconytė, G. (2015). Cartography of crime: Portrait of metropolitan Vilnius. Journal of Maps. Pp: 1-6. http://doi.org/10.1080/17445647.2015.1101404

Vozmediano, L. \& San Juan, C. (2006). Empleo de Sistemas de Información Geográfica en el estudio del Miedo al Delito. Revista Española de Investigación Criminológica: REIC, No. 4.

Vozmediano L., \& San Juan C. (2011). Criminología Ambiental: ecología del delito y de la seguridad. Editorial UOC.

Wheeler, A. P., Worden, R. E., \& McLean, S. J. (2015). "Replicating group-based trajectory models of crime at micro-places in Albany, NY". Journal of Quantitative Criminology, pp: 1-24. http://doi.org/10.1007/ s10940-015-9268-3

Wortley, R., \& Mazerolle, L. (Eds.). (2013). Environmental criminology and crime analysis. Willan. 